\title{
Stability of Toroidal Magnetic Fields in the Radiation Zone of a Star
}

\author{
L. L. Kitchatinov \\ Institute of Solar-Terrestrial Physics, Siberian Branch, Russian Academy of Sciences, \\ P.O. Box 4026, Irkutsk, 664033 Russia \\ Received April 4, 2007; in final form, June 22, 2007
}

\begin{abstract}
The stability of a toroidal magnetic field in the rotating radiation zone of a star is analyzed to estimate the maximum possible magnitude of relic fields. Equations for small perturbations are obtained taking into account the finite diffusivity and the stabilizing effect of the subadiabatic stratification. The numerical solution of the eigenvalue problem indicates that the threshold field strength for the onset of instability in the radiation zone of the Sun is about 600 G. This figure sets an upper bound for the strength of the relic field. The assumption that magnetic instabilities are present in the solar radiation zone disagrees with the observed abundance of lithium. Our analysis of joint stability of toroidal field and nonuniform rotation shows that two-dimensional MHD solutions for the solar tachocline are stable against threedimensional perturbations.

PACS numbers: $52.30 . \mathrm{Cv}, 96.60 . \mathrm{Hv}, 97.10 . \mathrm{Kc}, 96.60 . \mathrm{Bn}, 96.60 . \mathrm{Jw}$

DOI: $10.1134 / \mathrm{S} 1063772908030074$
\end{abstract}

\section{INTRODUCTION}

Stellar magnetic activity is generally attributed to the action of a hydromagnetic dynamo in stellar convection zones. The magnetic fields localized in stably stratified radiation zones can, however, also be very important - in particular, for angular-momentum transport in the stellar interior [1,2]. The fields of early-type stars are coupled with their outer radiation zones. A weak poloidal field in the radiation zone could be responsible for the formation of the solar tachocline [3]. Strong toroidal fields can affect the g-modes of global oscillations [4] and solar neutrinos [5].

To all appearances, a hydromagnetic dynamo cannot operate in the radiation zone, where no flows with energies sufficiently high for a dynamo effect are present. However, the Ohmic-dissipation times for magnetic fields in the radiation zones reach several billion years [6]. Therefore, relic magnetic fields acquired by the star at early stages of its evolution can persist there. However, it is not clear how strong such fields can be. The radiation zone of the Sun is located deep $\left(r \leq 0.713 R_{\odot}\right.$, where $R_{\odot}$ is the solar radius [7]) below the solar surface, so that possibilities for measuring the magnetic fields there are limited (see, however, [8]). Direct calculations of relic fields $[9,10]$ are based on many assumptions, and it is not entirely clear how well these are substantiated. We investigate here a stability analysis of the magnetic field. To persist in the radiation zone on stellarevolution time scales, the relic field must be stable.
Therefore, the threshold strength for the development of instabilities seems to set an upper limit to the relicfield strength. Stability issues are also important for the theory of the solar tachocline [11] and the problem of the transport of angular momentum and chemical admixtures in the radiation zones of stars [12].

Our study is restricted to the case of an axisymmetric toroidal, or azimuthal, magnetic field. The toroidal component is normally the largest one when even a small rotational nonuniformity is present. Note that theoretical estimates of possible poloidal field strengths are not so important. Poloidal fields (if they are not too weak [3]) can penetrate from the radiative zone through the convective zone to the solar surface, and can therefore be estimated from observations [13]. Toroidal fields do not possess this property, so that theoretical considerations must be invoked to estimate them.

Among various instabilities that can develop in the rotating radiation zone of a star with a toroidal field [14], the kink instability of pinches [15] appears to be most important [16]. This is because this instability proceeds via nearly horizontal displacements (the meaning of the word "nearly" here will be explained in Section 2.1). In the sub-adiabatically stratified radiation zones, radial displacements are inhibited by buoyant forces.

This instability was studied previously using the energy principle for a nonrotating medium in the absence of dissipation [17, 18]. However, it later became clear that rotation and finite thermal conductivity are 


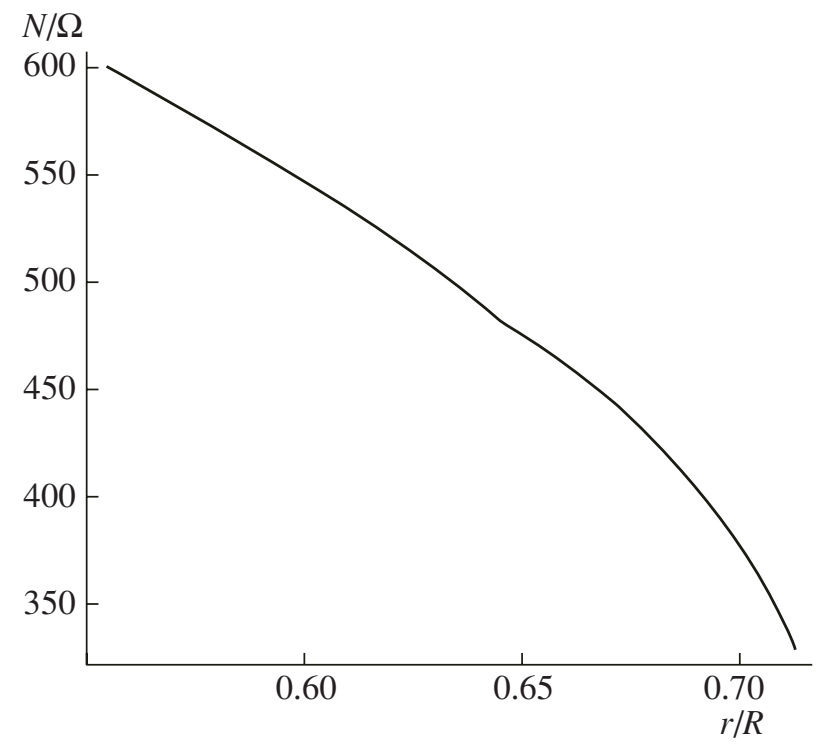

Fig. 1. Ratio of the internal gravity-wave frequency (1) to the angular velocity in the upper region of the solar radiation zone as a function of the relative heliocentric radius. This dependence was obtained from the model for the internal structure of the Sun [19].

important [16]. In particular, this follows from the instability analysis of Acheson [14] in a local (smallscale) approximation. However, it follows from [14] that the threshold field strengths at which the instability sets in decrease with increasing horizontal scale of the perturbations.

Here, we take into account both diffusion and rotation. We consider perturbations that are global in horizontal directions but have a small radial scale. As we will demonstrate, precisely such a scale ratio is typical of most unstable perturbations (represented by radially thin layers encompassing the entire radiative zone in horizontal directions). We will see that small radial displacements are important, since the instability disappears in the (two-dimensional) approximation of purely horizontal displacements. An estimate of the effective diffusion of chemical species due to radial mixing shows that the toroidal field in the radiation zone of the Sun cannot exceed the instability threshold $(\simeq 600 \mathrm{G})$ by a significant amount: otherwise, this would lead to contradictions with lithiumabundance data. We will also consider the stability of the coupled toroidal field and differential rotation. Our results indicate that axisymmetric solutions for the solar tachocline [3] are stable against nonaxisymmetric perturbations.

\section{FORMULATION OF THE PROBLEM}

\subsection{Background State and Basic Assumptions}

Consider the radiative zone of a star with stable (sub-adiabatic) stratification and, accordingly, a pos- itive Brunt-Väisälä frequency:

$$
N^{2}=\frac{g}{c_{\mathrm{p}}} \frac{\partial s}{\partial r}
$$

Here, $s=c_{\mathrm{p}} \ln \left(P / \rho^{\gamma}\right)$ is the specific entropy. We assume that the medium rotates at an angular velocity $\Omega(r, \theta)$, which can be position-dependent. We use here the usual spherical coordinates $r, \theta$, and $\phi$. An azimuthal magnetic field

$$
\mathbf{B}=\mathbf{e}_{\phi} r \sin \theta \sqrt{4 \pi \rho} \Omega_{\mathrm{A}}(r, \theta)
$$

is also present; here, $\Omega_{\mathrm{A}}$ is the angular Alfvén frequency and $\mathbf{e}_{\phi}$ is the unit vector in the azimuthal direction.

Generally, the rotation and magnetic field break down the spherical symmetry. The azimuthal component of the equation for the vorticity rotu (where $\mathbf{u}$ is the velocity) yields

$$
\begin{gathered}
r \sin \theta \frac{\partial\left(\Omega_{\mathrm{A}}^{2}-\Omega^{2}\right)}{\partial z} \\
=\frac{1}{\rho^{2}}\left[\nabla \rho \times \nabla\left(P+\frac{B^{2}}{8 \pi}\right)\right]_{\phi},
\end{gathered}
$$

where the contribution of the viscosity is omitted and $\frac{\partial}{\partial z}=\cos \theta \frac{\partial}{\partial r}-r^{-1} \times \sin \theta \frac{\partial}{\partial \theta}$ is the spatial derivative in the direction of the rotational axis. Therefore, the pressure and density in the spherical coordinate system depend not only on the radius, but also on the latitude, and there is no coordinate system in which the thermodynamic parameters depend on only one variable. Nevertheless, if the rotation is slow and the magnetic field is weak, so that

$$
\Omega \ll N, \quad \Omega_{\mathrm{A}} \ll N,
$$

departures from spherical symmetry are small, and we will neglect them, assuming that the thermodynamic parameters depend only on the radius $r$. The ratio $N / \Omega$ for the solar radiative zone is plotted in Fig. 1. Note that $\Omega_{\mathrm{A}}$ for the upper part of the solar radiative zone reaches the $\Omega$ value for a field strength of $B \sim$ $10^{5} \mathrm{G}$.

The quantity $N / \Omega$ is also an estimate of the ratio of the radial and meridional velocities, $u_{r}^{\prime} / u_{\theta}^{\prime} \sim$ $\Omega^{2} / N^{2}[20]$ (here and below, primes mark small perturbations of the background state). For this reason, radial velocities are often neglected. However, this substantially narrows the class of possible perturbations, as can be seen from the expression for solenoidal velocity perturbations in a spherical geometry expressed in terms of the scalar potentials of the poloidal $\left(P_{\mathrm{V}}\right)$ and toroidal $\left(T_{\mathrm{V}}\right)$ flows $[21]$ :

$$
\mathbf{u}^{\prime}=\frac{\mathbf{e}_{r}}{r^{2}} \hat{L} P_{\mathrm{v}}-\frac{\mathbf{e}_{\theta}}{r}\left(\frac{1}{\sin \theta} \frac{\partial T_{\mathrm{V}}}{\partial \phi}+\frac{\partial^{2} P_{\mathrm{V}}}{\partial r \partial \theta}\right)
$$




$$
\begin{gathered}
+\frac{\mathbf{e}_{\phi}}{r}\left(\frac{\partial T_{\mathrm{V}}}{\partial \theta}-\frac{1}{\sin \theta} \frac{\partial^{2} P_{\mathrm{V}}}{\partial r \partial \phi}\right), \\
\hat{L}=\frac{1}{\sin \theta} \frac{\partial}{\partial \theta} \sin \theta \frac{\partial}{\partial \theta}+\frac{1}{\sin ^{2} \theta} \frac{\partial^{2}}{\partial \phi^{2}} .
\end{gathered}
$$

The assumption that $u_{r}=0$ completely eliminates poloidal velocity perturbations. The horizontal component of the poloidal flow can, however, remain constant as the radial velocity is reduced, if the vertical scale of the perturbations is proportionally decreased. As we will see, no kink instability [18] is present at $u_{r}=0$. At the same time, this instability can develop in the presence of very small vertical displacements, $u_{r} \ll u_{\theta}$ (even arbitrarily small in the absence of dissipation), if the vertical scales are also small, $k r \gg 1$ (here, $k$ is the vertical wavenumber). In this sense, the displacements in unstable perturbations are indeed nearly horizontal.

Our stability analysis is local (small-scale) in radius, i.e., the radial dependence of the perturbations is assumed to have the form $\exp (i k r)$. At the same time, the modes with the largest horizontal scales are the first to lose their stability [16], so that the analysis remains global in the horizontal directions. Subsequent calculations will confirm that the unstable perturbations comply with these restrictions.

Since the radial scales are small, the perturbations can be considered incompressible: $\operatorname{div} \mathbf{u}=0$ [14]. Therefore, the representation (5) for the velocity perturbations can be used together with a similar formula for magnetic perturbations:

$$
\begin{gathered}
\mathbf{B}^{\prime}=\frac{\mathbf{e}_{r}}{r^{2}} \hat{L} P_{\mathrm{m}}-\frac{\mathbf{e}_{\theta}}{r}\left(\frac{1}{\sin \theta} \frac{\partial T_{\mathrm{m}}}{\partial \phi}+\frac{\partial^{2} P_{\mathrm{m}}}{\partial r \partial \theta}\right) \\
+\frac{\mathbf{e}_{\phi}}{r}\left(\frac{\partial T_{\mathrm{m}}}{\partial \theta}-\frac{1}{\sin \theta} \frac{\partial^{2} P_{\mathrm{m}}}{\partial r \partial \phi}\right) .
\end{gathered}
$$

The following assumption refers to pressure perturbations. We will neglect the local pressure perturbations. Note that we will use the equation for vorticity $\boldsymbol{\omega}=\operatorname{rot}^{\prime}$. To this end, we take the curl of the equation of motion; therefore, pressure perturbations due to nonlinear terms in the equation of motion are automatically included. We assume that the local pressure is determined by hydrostatic equilibrium, and that thermal perturbations develop under constant pressure, i.e., $\rho^{\prime} / \rho=-T^{\prime} / T=-s^{\prime} / c_{\mathrm{p}}$. A different interpretation of this assumption is given in [14], where the perturbations of the total pressure (including the magnetic contribution) were neglected. In this case, an instability due to magnetic buoyancy sets in. In our approach, no such instability is present (it appears in terms that are one order higher than the contributions taken into account in our equations used below, in terms of the vertical-tohorizontal scale ratio of the perturbations), so that the assumptions of constant total pressure and constant gas pressure are equivalent.

\subsection{Perturbation Equations}

The original system of equations linearized with respect to small perturbations includes equations for perturbations of the velocity,

$$
\begin{gathered}
\frac{\partial \mathbf{u}^{\prime}}{\partial t}+(\mathbf{u} \cdot \nabla) \mathbf{u}^{\prime}+\left(\mathbf{u}^{\prime} \cdot \nabla\right) \mathbf{u} \\
+\left[\nabla\left(\mathbf{B} \cdot \mathbf{B}^{\prime}\right)-(\mathbf{B} \cdot \nabla) \mathbf{B}^{\prime}-\left(\mathbf{B}^{\prime} \cdot \nabla\right) \mathbf{B}\right] / 4 \pi \rho \\
=-\left(\frac{1}{\rho} \nabla P\right)^{\prime}+\nu \Delta \mathbf{u}^{\prime},
\end{gathered}
$$

magnetic field,

$$
\frac{\partial \mathbf{B}^{\prime}}{\partial t}=\nabla \times\left(\mathbf{u} \times \mathbf{B}^{\prime}+\mathbf{u}^{\prime} \times \mathbf{B}-\eta \nabla \times \mathbf{B}^{\prime}\right)
$$

and entropy,

$$
\frac{\partial s^{\prime}}{\partial t}+\mathbf{u} \cdot \nabla s^{\prime}+\mathbf{u}^{\prime} \cdot \nabla s=\frac{c_{\mathrm{p}} \chi}{T} \Delta T^{\prime} .
$$

These equations already take into account the fact that the vertical-variation scale of these perturbations is small compared to the variation scale of the background state. The unperturbed flow is uniform rotation with angular velocity $\Omega(r, \theta)$, and the unperturbed magnetic field, defined in (2), is toroidal.

In the perturbation equations, we retain only terms of the lowest order in the ratio of the vertical perturbation scale to the radius $r$; i.e., we use an approximation local in $r$. This enables us to write the radial (and also azimuthal and temporal) dependence of the perturbations as $\exp (-i \omega t+i m \phi+i k r)$. Instability will develop if an eigenvalue $\omega$ with a positive imaginary part is present.

To describe the velocity and magnetic-field perturbations, we use the scalar potentials introduced in (5) and (6). The radial components of the curl of (7) and (8) yield equations for the toroidal flow and toroidal magnetic field, respectively. The radial component of (8) leads to an equation for the poloidal magnetic field. An equation for the poloidal flow can be obtained by applying the curl operation twice to (7) and taking the radial component. When this is done, the contribution of the pressure assumes the form

$$
\begin{gathered}
\mathbf{r} \cdot \nabla \times \nabla \times\left(\frac{1}{\rho} \nabla P\right)^{\prime} \\
=-\mathbf{r} \cdot \nabla \times\left(\frac{1}{\rho^{2}}(\nabla \rho) \times(\nabla P)\right)^{\prime} \\
=\frac{\mathbf{r}}{c_{\mathrm{p}}} \cdot \nabla \times\left(\frac{1}{\rho}(\nabla s) \times(\nabla P)\right)^{\prime}
\end{gathered}
$$




$$
=-\frac{\mathbf{r}}{c_{\mathrm{p}}} \cdot \nabla \times\left(\mathbf{g} \times\left(\nabla s^{\prime}\right)\right)=\frac{g}{r c_{\mathrm{p}}} \hat{L} s^{\prime} .
$$

We now reformulate the relations in terms of dimensionless quantities, measuring time in units of $\Omega_{0}^{-1}$ (where $\Omega_{0}$ is the angular velocity at the equator) and velocity in units of $r \Omega_{0}$, and introduce the dimensionless eigenvalues $\hat{\omega}=\omega / \Omega_{0}$. We also define the other dimensionless quantities as follows:

$$
\begin{gathered}
A=\frac{k}{\Omega_{0} r^{2} \sqrt{4 \pi \rho}} P_{\mathrm{m}}, \quad B=\frac{1}{\Omega_{0} r^{2} \sqrt{4 \pi \rho}} T_{\mathrm{m}}, \quad \\
V=\frac{k}{\Omega_{0} r^{2}} P_{\mathrm{v}}, \quad W=\frac{1}{\Omega_{0} r^{2}} T_{\mathrm{v}}, \quad S=\frac{i k r g}{c_{\mathrm{p}} r N^{2}} s^{\prime}, \\
\hat{\Omega}=\frac{\Omega}{\Omega_{0}}, \quad \hat{\Omega}_{\mathrm{A}}=\frac{\Omega_{\mathrm{A}}}{\Omega_{0}} .
\end{gathered}
$$

Here, the coefficient $k r$ is included in the definitions of the poloidal-field potentials $V$ and $A$ to give them the same order as the toroidal-field potentials $W$ and $B$.

The equation for the poloidal (perturbation) flow is

$$
\begin{gathered}
\hat{\omega} \hat{L} V=-\hat{\lambda}^{2} \hat{L} S-i \frac{\epsilon_{\nu}}{\hat{\lambda}^{2}}(\hat{L} V)-2 \mu \hat{\Omega} \hat{L} W \\
-2\left(1-\mu^{2}\right) \frac{\partial(\mu \hat{\Omega})}{\partial \mu} \frac{\partial W}{\partial \mu}-2 m^{2} \frac{\partial \hat{\Omega}}{\partial \mu} W \\
+2 \mu \hat{\Omega}_{\mathrm{A}} \hat{L} B+2\left(1-\mu^{2}\right) \frac{\partial\left(\mu \hat{\Omega}_{\mathrm{A}}\right)}{\partial \mu} \frac{\partial B}{\partial \mu} \\
+2 m^{2} \frac{\partial \hat{\Omega}_{\mathrm{A}}}{\partial \mu} B-m \hat{\Omega}_{\mathrm{A}} \hat{L} A-2 m \frac{\partial\left(\mu \hat{\Omega}_{\mathrm{A}}\right)}{\partial \mu} A \\
-2 m\left(1-\mu^{2}\right) \frac{\partial \hat{\Omega}_{\mathrm{A}}}{\partial \mu} \frac{\partial A}{\partial \mu}+m \hat{\Omega} \hat{L} V \\
+2 m \frac{\partial(\mu \hat{\Omega})}{\partial \mu} V+2 m\left(1-\mu^{2}\right) \frac{\partial \hat{\Omega}}{\partial \mu} \frac{\partial V}{\partial \mu}
\end{gathered}
$$

where $\mu=\cos \theta$, and the quantity

$$
\hat{\lambda}=\frac{N}{\Omega_{0} k r}
$$

can be regarded as the special normalization for vertical wavelength of the perturbation. We can see that the Brunt-Väisälä frequency $N(1)$ appears only in the first term of the right-hand side of (12). Therefore, the stabilizing effect of the buoyant force weakens as the vertical scale of the perturbations is reduced. It is for this reason that modes with large $k r$ are most unstable. The diffusion parameter $\epsilon_{\nu}$ and also the magnetic-diffusion and thermal-diffusivity parameters $\epsilon_{\eta}$ and $\epsilon_{\chi}$ (which will be used below) are defined as

$$
\epsilon_{\nu}=\frac{\nu N^{2}}{\Omega_{0}^{3} r^{2}}, \quad \epsilon_{\eta}=\frac{\eta N^{2}}{\Omega_{0}^{3} r^{2}}, \quad \epsilon_{\chi}=\frac{\chi N^{2}}{\Omega_{0}^{3} r^{2}} ;
$$

therefore, the frequency $N$ cancels in the second term.

Apart from (12), the full system includes four other equations. These are the equations for the toroidal flow,

$$
\begin{gathered}
\hat{\omega} \hat{L} W=-i \frac{\epsilon_{\nu}}{\hat{\lambda}^{2}} \hat{L} W+m \hat{\Omega} \hat{L} W \\
-m \hat{\Omega}_{\mathrm{A}} \hat{L} B-m W \frac{\partial^{2}}{\partial \mu^{2}}\left[\left(1-\mu^{2}\right) \hat{\Omega}\right] \\
+m B \frac{\partial^{2}}{\partial \mu^{2}}\left[\left(1-\mu^{2}\right) \hat{\Omega}_{\mathrm{A}}\right]+(\hat{L} V) \\
\times \frac{\partial}{\partial \mu}\left[\left(1-\mu^{2}\right) \hat{\Omega}\right]-(\hat{L} A) \frac{\partial}{\partial \mu}\left[\left(1-\mu^{2}\right) \hat{\Omega}_{\mathrm{A}}\right] \\
+\left\{\frac{\partial}{\partial \mu}\left[\left(1-\mu^{2}\right)^{2} \frac{\partial \hat{\Omega}}{\partial \mu}\right]-2\left(1-\mu^{2}\right) \hat{\Omega}\right\} \frac{\partial V}{\partial \mu} \\
-\left\{\frac{\partial}{\partial \mu}\left[\left(1-\mu^{2}\right)^{2} \frac{\partial \hat{\Omega}_{\mathrm{A}}}{\partial \mu}\right]-2\left(1-\mu^{2}\right) \hat{\Omega}_{\mathrm{A}}\right\} \frac{\partial A}{\partial \mu}
\end{gathered}
$$

the toroidal magnetic field,

$$
\begin{gathered}
\hat{\omega} \hat{L} B=-i \frac{\epsilon_{\eta}}{\hat{\lambda}^{2}} \hat{L} B+m \hat{L} \hat{\Omega} B-m \hat{L} \hat{\Omega}_{\mathrm{A}} W \\
-m^{2} \frac{\partial \hat{\Omega}}{\partial \mu} A-\frac{\partial}{\partial \mu}\left[\left(1-\mu^{2}\right)^{2} \frac{\partial \hat{\Omega}}{\partial \mu} \frac{\partial A}{\partial \mu}\right] \\
+m^{2} \frac{\partial \hat{\Omega}_{\mathrm{A}}}{\partial \mu} V+\frac{\partial}{\partial \mu}\left[\left(1-\mu^{2}\right)^{2} \frac{\partial \hat{\Omega}_{\mathrm{A}}}{\partial \mu} \frac{\partial V}{\partial \mu}\right] ;
\end{gathered}
$$

the poloidal field,

$$
\hat{\omega} \hat{L} A=-i \frac{\epsilon_{\eta}}{\hat{\lambda}^{2}} \hat{L} A+m \hat{\Omega} \hat{L} A-m \hat{\Omega}_{\mathrm{A}} \hat{L} V ;
$$

and the entropy perturbations,

$$
\hat{\omega} S=-i \frac{\epsilon_{\chi}}{\hat{\lambda}^{2}} S+m \hat{\Omega} S+\hat{L} V .
$$

In the absence of rotation and magnetic field $(\Omega=$ $\Omega_{\mathrm{A}}=0$ ), then, neglecting diffusion, we obtain the eigenvalues spectrum

$$
\omega=\frac{N}{k r} \sqrt{l(l+1)}, \quad l=1,2,3, \ldots,
$$

which correspond to stable internal gravitational waves (g modes). Therefore, the instabilities that will subsequently be revealed are due to the magnetic field and/or nonuniform rotation.

\subsection{Two-Dimensional Approximation}

The ratio $N / \Omega$ can be so large in stellar radiation zones (Fig. 1) that the parameter $\hat{\lambda}$ (13) can be large despite the inequality $k r \gg 1$. When $\hat{\lambda} \gg 1$, to lowest order in the perturbation theory in $\hat{\lambda}^{-2}$, (12) yields

ASTRONOMY REPORTS Vol.52 No.3 2008 
$S=0$. It then follows from (18) that $V=0$, and (17) yields $A=0$. Thus, we arrive at the following system of equations for the toroidal perturbations:

$$
\begin{gathered}
\hat{\omega} \hat{L} W=m \hat{\Omega} \hat{L} W-m \hat{\Omega}_{\mathrm{A}} \hat{L} B \\
-m W \frac{\partial^{2}}{\partial \mu^{2}}\left[\left(1-\mu^{2}\right) \hat{\Omega}\right]+m B \frac{\partial^{2}}{\partial \mu^{2}}\left[\left(1-\mu^{2}\right) \hat{\Omega}_{\mathrm{A}}\right] \\
\hat{\omega} \hat{L} B=m \hat{L} \hat{\Omega} B-m \hat{L} \hat{\Omega}_{\mathrm{A}} W .
\end{gathered}
$$

These equations were derived in a different way in [22]. Here, they emerge as a limiting case in a more general approach. Equations (20) of the twodimensional approximation describe perturbations on isolated spherical surfaces. They have been widely used to analyze the stability of the solar tachocline. As we will see, the condition $\hat{\lambda} \lesssim 1$ is satisfied for the least stable perturbations. The two-dimensional approximation overestimates the critical values of the parameters for the onset of instabilities and, in all likelihood, overlooks the Vandakurov-Tayler magnetic instability $[17,18]$ (i.e., the kink instability [15] in the radiation zone of the star).

This can be ascertained by considering the case of uniform rotation and a constant Alfvén frequency ( $\Omega=$ const and $\Omega_{\mathrm{A}}=$ const). The latter condition implies that $B_{\phi} \sim \sin \theta$, and the Vandakurov-Tayler instability should be present [23]. Indeed, direct computations based on the equations of Section 2.2 show this to be the case [24]. However, the twodimensional approximation exhibits only stable solutions. When $\Omega$ and $\Omega_{\mathrm{A}}$ are constant, Eqs. (20) have constant coefficients and admit an analytical solution. We assume that $W, B \sim P_{l}^{m}(\mu)$ (where $P_{l}^{m}(\mu)$ are the associated Legendre polynomials) to find the eigenvalue spectrum

$$
\begin{gathered}
\frac{\hat{\omega}}{m}=1-\frac{1}{l(l+1)} \\
\pm \sqrt{\hat{\Omega}_{\mathrm{A}}^{2}\left[1-\frac{2}{l(l+1)}\right]+\frac{1}{l^{2}(1+l)^{2}}}
\end{gathered}
$$

which represents (stable) $r$ modes $[25,26]$ modified by the magnetic field.

The absence of magnetic instability in the twodimensional approximation has been demonstrated only for the special case of constant $\Omega$ and $\Omega_{\mathrm{A}}$, but, to all appearances, this remains valid for arbitrary $\Omega_{\mathrm{A}}(\mu)$ profiles. This can be seen from the following qualitative considerations. The magnetic fields in the case at hand can be represented as a set of closed magnetic flux tubes. Incompressible perturbations do not change the volume of the tubes but can change their length, and the magnetic energy of a tube varies in proportion to its length [27]. Therefore, due to the release of magnetic energy, the excitation of instability requires shortening of the tubes, which is not possible in the two-dimensional approximation for the following reason. The area of a segment of a spherical surface encircled by closed toroidal-field lines remains constant under two-dimensional incompressible deformations. The lengths of the circular lines of the background toroidal field are minimum for the given encircled area. Therefore, two-dimensional deformations can only increase the length of the magnetic flux tubes and, accordingly, the magnetic energy. Thus, it becomes clear that radial displacements, i.e., threedimensional perturbations, are necessary to excite the instability considered. Only three-dimensional perturbations can give rise to an instability that entails a release of magnetic energy.

\subsection{Parameters of the Problem}

We present here our results for particular nonuniform profiles for the rotation,

$$
\hat{\Omega}=1-a \cos ^{2} \theta,
$$

and the magnetic field,

$$
\hat{\Omega}_{\mathrm{A}}=b \cos \theta .
$$

Formula (22) provides a good approximation for the differential rotation in the solar tachocline [28]. Formula (23) describes a distribution of the toroidal field that is antisymmetric about the equator. Such a field structure is generated from a dipolar poloidal field by differential rotation. The parameters $a$ and $b$ determine the degree of nonuniformity of the rotation and the amplitude of the toroidal field.

A finite diffusivity is very important for stability, not only due to the small vertical scales, but mainly because the finite thermal conductivity reduces the stabilizing effect of the buoyant forces. The results discussed below were obtained for the following values of the diffusion parameters (14), which correspond to the upper radiation zone of the Sun:

$$
\begin{gathered}
\epsilon_{\chi}=10^{-4}, \quad \epsilon_{\eta}=4 \times 10^{-8} \\
\epsilon_{\nu}=2 \times 10^{-10} .
\end{gathered}
$$

The eigenvalue problem for (12) and (15)-(18) was solved numerically.

\section{RESULTS \\ 3.1. Uniform Rotation}

Below the tachocline, the rotation of the solar radiative zone is nearly uniform, and we can assume that $a=0$ in (22) for this region. This case is also interesting because the uniform rotation has no destabilizing effect, and the magnetic instability can be considered in a pure form. 


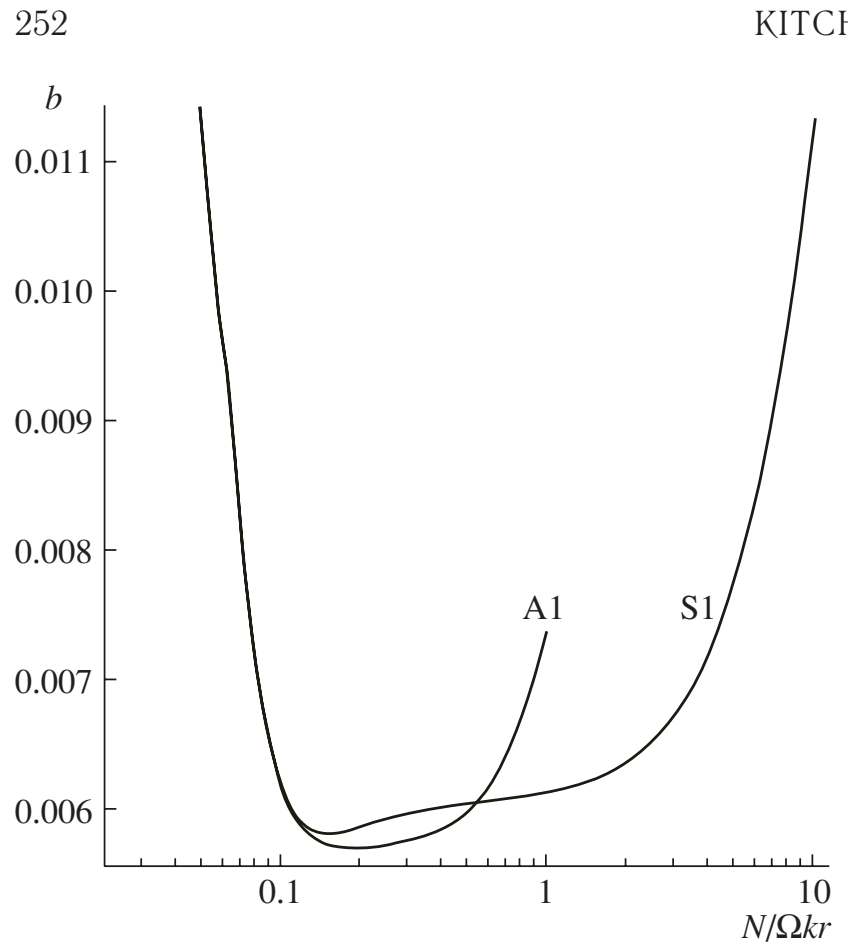

Fig. 2. Boundaries of the stability region for symmetric (S1) and antisymmetric (A1) perturbations. The instability is present in the region above the curves.

Figure 2 shows a stability diagram. There are two classes of perturbations with different symmetries about the equator. For the perturbations that we will call symmetric and denote $\mathrm{S} m$ (where $m$ is the azimuthal wavenumber), the potentials $W$ and $A$ are symmetric about the equator, while the entropy perturbation and the potentials $B$ and $V$ are antisymmetric. The perturbations with the opposite symmetry are denoted $\mathrm{Am}$. The instability is manifest only for non-axisymmetric perturbations with $m=1$.

The instability sets in as the magnetic field exceeds its critical value $b_{\mathrm{c}} \simeq 6 \times 10^{-3}$. The perturbations for which $\hat{\lambda}=N / \Omega_{0} k r \sim 0.1$ lose their stability first. Since $N / \Omega$ is large (Fig. 1), this confirms that the unstable perturbations, which are global in the horizontal directions, have a small vertical scale.

We can see from Fig. 3 that the range of radial scales of the unstable perturbations widens with increasing field strength, although the maximum growth rates are reached near $\hat{\lambda} \sim 0.1$. As $\hat{\lambda}$ is increased, the growth rates approach zero, while they can even become negative as it is decreased. To all appearances, this is related to diffusion. The growth rates of the unstable perturbations are not large. The doubling time for the perturbations can become less than the rotational period only for strong fields with $\Omega_{\mathrm{A}}>\Omega(b>1)[24]$.

The released magnetic energy can feed the instability only if the perturbations shorten the magnetic flux tubes (see the final part of Section 2.3). Such shortening is clearly visible in Fig. 4.

\subsection{Instability of the Coupled Magnetic Field and Nonuniform Rotation}

Figures 5 and 6 present the growth rates of the unstable perturbations when both a magnetic field and differential rotation are present, as is believed to characterize the solar tachocline. The differential rotation by itself can result in instability even without a magnetic field. In the two-dimensional approximation, such an instability sets in when $a>0.28$ [20]. This is reflected by the nearly horizontal line for $\hat{\sigma}=$ $10^{-2}$ in Fig. 5 ( $\hat{\sigma}=\frac{\operatorname{Im} \omega}{\Omega_{0}}$ is the dimensionless growth rate). The critical value of $a$ for three-dimensional perturbations is reduced to $a=0.21$ [24]. As $a$ is further decreased to $a \simeq 0.1$, and also for S1 perturbations, no hydrodynamic instability is present; however, an arbitrarily weak magnetic field gives rise to instability. In this case, $\hat{\sigma} \sim b$.

When $a<0.1$, the instability is manifest if the magnetic field exceeds a critical value, $b_{\mathrm{c}}>0.002$. The magnetic instabilities present in the region of hydrodynamic stability have relatively small growth rates.

When the differential rotation is sufficiently strong, $a>0.39$, a hydrodynamic instability of S2 perturbations is also present [24], which persists in the presence of a magnetic field. However, the growth rates of S2 perturbations are small compared to those for the A1 and S1 modes, so that we do not present the results for those perturbations. We also do not consider instabilities that are manifest in the case of an "antisolar" differential rotation with $a<0$ (relatively fast rotation at the poles).

\section{DISCUSSION}

Returning to dimensional quantities, we find that the vertical scale of the perturbations in the upper part of the solar radiation zone is $\lambda \simeq 10 \hat{\lambda} \mathrm{Mm}$, and

$$
B_{\phi} \simeq 10^{5} b \mathrm{G} \text {. }
$$

Therefore, in the region below the tachocline, the magnetic instability develops on vertical scales of the order of $1000 \mathrm{~km}$, while the threshold strength of the field is about $600 \mathrm{G}$ (Fig. 2). In all likelihood, this figure represents an upper limit for the relic field of the Sun. Stronger fields will be destroyed by the instability.

ASTRONOMY REPORTS Vol.52 No.3 2008 


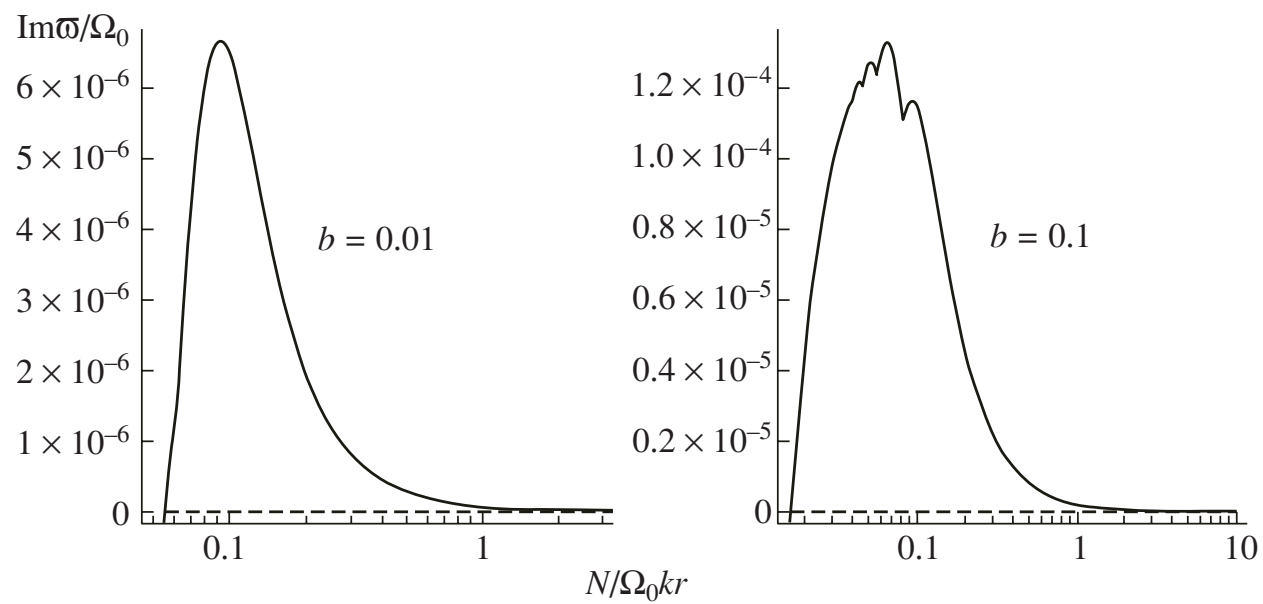

Fig. 3. Growth rates of the most rapidly growing perturbations as a function of their vertical scale $\hat{\lambda}$ [see (13)] for two values of the magnetic-field amplitude $b$.
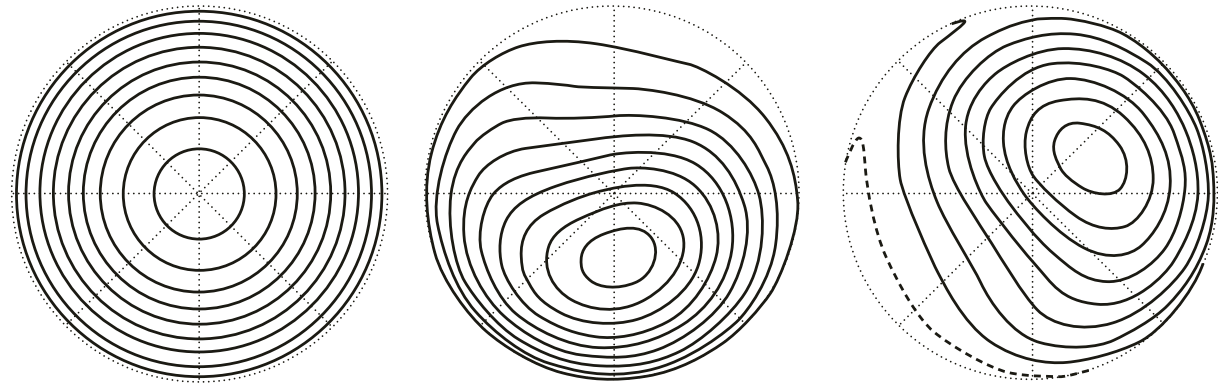

Fig. 4. Pattern of the toroidal magnetic field viewed from the pole: unperturbed field (left) and the field superposed with the most rapidly growing perturbations with equatorial symmetry types S1 (center) and A1 (right), for $b=0.1$. The shortening of the magnetic flux tubes with the development of the instability can be seen. The global rotation is clockwise (south pole). The azimuthal drift of the unstable perturbations is opposite to this direction.

It has been suggested, however, that instability of the toroidal field in the presence of weakly nonuniform rotation can amplify, rather than destroy, the magnetic field [29]. This possibility results from the presence of radial displacements in the unstable perturbations, which transform the toroidal field into a poloidal field. This poloidal field is again transformed into a toroidal field by the nonuniform rotation, which can close the field-amplification cycle.

However, radial mixing must satisfy certain constraints related to available data on the chemical composition of the Sun. Light elements, such as lithium, beryllium, and boron, become depleted in the stellar interior at temperatures exceeding some critical value [12]. In particular, lithium catalyzes and is rapidly destroyed by nuclear reactions at temperatures over $2.7 \times 10^{6} \mathrm{~K}$, which corresponds to depths of $r \leq 0.65 R_{\odot}$ for the Sun ${ }^{1)}$. Therefore, the effective diffusion coefficient $D_{\mathrm{T}}$ in the upper part of the solar radiation zone cannot substantially exceed $10^{3} \mathrm{~cm}^{2} / \mathrm{s}[12,30]$.

We will use the usual estimate $D_{\mathrm{T}} \simeq u_{r}^{\prime} \ell$, where $u_{r}^{\prime}$ and $\ell$ are the rms speed and characteristic scale of the radial mixing, respectively. The mixing speed can be estimated by equating the growth rate of the perturbations, $\sigma=\operatorname{Im} \omega$, to the characteristic mixing rate: $\sigma \simeq u_{r}^{\prime} / \ell$ (note that, for the instability considered, the characteristic mixing times in the horizontal and radial directions coincide, i.e., $\left.r / u_{\theta}^{\prime} \simeq \ell / u_{r}^{\prime}\right)$. Setting $\ell=\lambda / 2$ and taking into account the fact that the growth rates of the perturbations reach their

\footnotetext{
${ }^{1)}$ The critical temperatures at which beryllium and boron are depleted are $3.5 \times 10^{6} \mathrm{~K}$ and $5 \times 10^{6} \mathrm{~K}$, and are reached at depths of $r=0.54 R_{\odot}$ and $r=0.4 R_{\odot}$, respectively.
} 


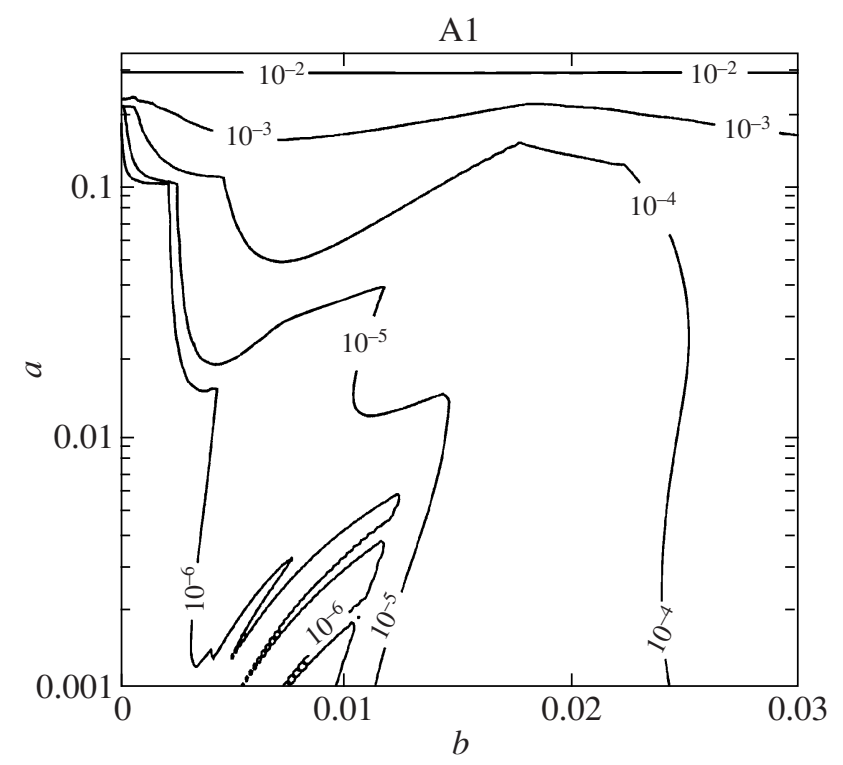

Fig. 5. Contours of the growth rates of the unstable perturbations with equatorial symmetry type A1 in the plane of the parameters $a$ and $b$, which determine the nonuniformity of rotation and the magnetic-field strength, respectively. The contours are labeled with values of the dimensionless growth rate $\hat{\sigma}=\frac{\operatorname{Im} \omega}{\Omega_{0}}$.

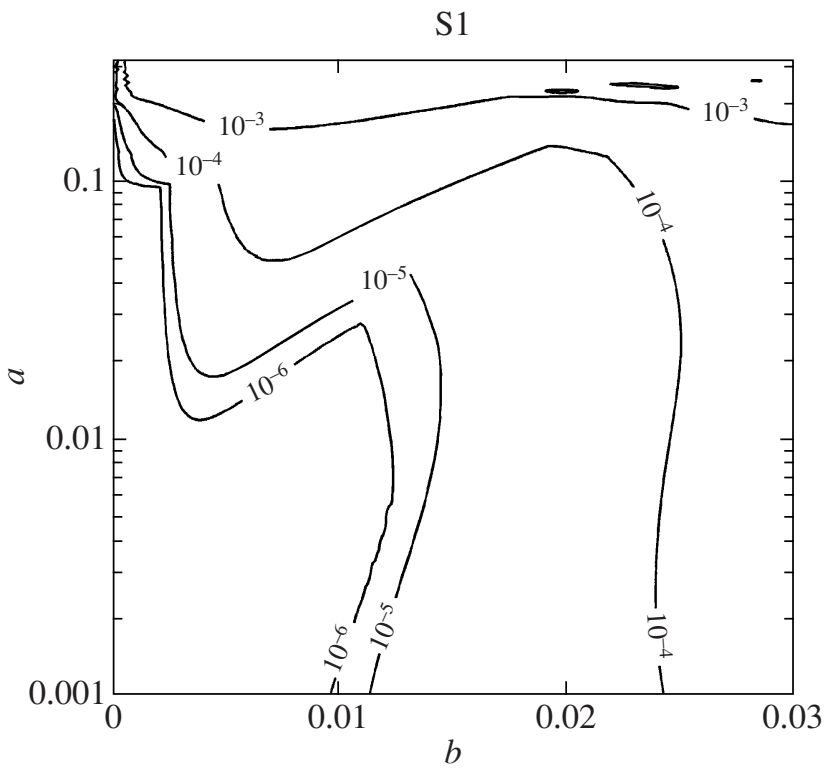

Fig. 6. Same as Fig. 5 for perturbations with equatorial symmetry type S1.

maximum at $\hat{\lambda} \simeq 0.1$ (see Fig. 3), we obtain for the diffusion coefficient

$$
D_{\mathrm{T}} \simeq 7 \times 10^{9} \hat{\sigma} \mathrm{cm}^{2} / \mathrm{s},
$$

where we have $\hat{\sigma}=\sigma / \Omega_{0}$ for the normalized growth rate. The dependence of the diffusion coefficient on the magnetic field is presented in Fig. 7, which also shows the (dimensionless) growth rate of the perturbations. The dotted line in Fig. 7 corresponds to the approximation $\hat{\sigma}=0.1 b^{2}$, which leads to the following simple relationship between the diffusion coefficient and the strength of the toroidal field:

$$
D_{\mathrm{T}} \simeq 7 \times 10^{4}\left(\frac{B_{\phi}}{1 \mathrm{kG}}\right)^{2} \mathrm{~cm}^{2} / \mathrm{s} .
$$

The diffusion coefficient (Fig. 7) does not exceed $10^{3} \mathrm{~cm}^{2} / \mathrm{s}$.

Thus, we conclude that the strength of the toroidal field in the radiative zone cannot appreciably exceed 


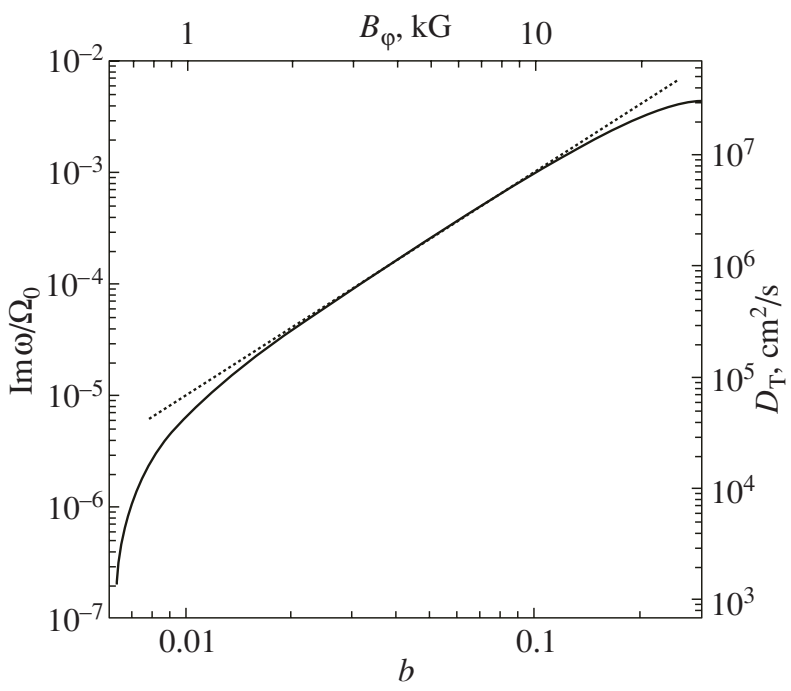

Fig. 7. Growth rate of unstable perturbations $\hat{\sigma}=\frac{\operatorname{Im} \omega}{\Omega_{0}}$ as a function of the parameter $b$ [see (23)] for $\hat{\lambda}=0.1$. The upper horizontal scale corresponds to the field strength calculated according to (25) and the right vertical scale to the effective diffusion coefficient for chemical species, according to (26). The dotted line represents the parabolic approximation $\hat{\sigma}=0.1 b^{2}$.

the critical value for the development of instability, which we have found to be about $600 \mathrm{G}$. Such a small magnetic field is not able to affect oscillations of the solar radiation zone or the solar neutrinos.

The results presented in Section 3.2 demonstrate that the two-dimensional axisymmetric solutions for the solar tachocline $[3,31]$ are stable with respect to non-axisymmetric perturbations.

Note that, since the radial scales of the unstable perturbations are small, the radial field can have a stabilizing effect, increasing somewhat the critical field strength. The inclusion of a poloidal field is one possible way in which this study can be further developed.

\section{ACKNOWLEDGMENTS}

This work was supported by the Russian Foundation for Basic Research (project no. 05-02-04015).

\section{REFERENCES}

1. P. Charbonneau and K. B. MacGregor, Astrophys. J. 387, 639 (1992).

2. G. Rüdiger and L. L. Kitchatinov, Astrophys. J. 466, 1078 (1996).

3. L. L. Kichatinov, Astron. Zh. 83, 1050 (2006)[Astron. Rep. 50, 944 (2006)].
4. T. I. Rashba, V. B. Semikoz, and J. W. F. Valle, Mon. Not. R. Astron. Soc. 370, 845 (2006).

5. P. A. Sturrock, G. Walther, and M. S. Wheatland, Astrophys. J. 507, 978 (1998).

6. T. G. Cowling, Mon. Not. R. Astron. Soc. 105, 166 (1945).

7. S. Basu and H. M. Antia, Mon. Not. R. Astron. Soc. 287, 189 (1997).

8. C. Burgess, N. S. Dzhaluilov, M. Maltoni, et al., Astrophys. J. 588, 65 (2003).

9. L. L. Kitchatinov, M. Jardine, and A. C. Cameron, Astron. Astrophys. 374, 250 (2001).

10. L. L. Kichatinov, Astron. Zh. 78, 934 (2001) [Astron. Rep. 45, 816 (2001)].

11. P. A. Gilman, Astron. Nachr. 326, 208 (2005).

12. G. Barnes, P. Charbonneau, and K. B. MacGregor, Astrophys. J. 511, 466 (1999).

13. E. E. Benevolenskaya and M. I. Pudovkin, Pis'ma Astron. Zh. 8, 506 (1982) [Sov. Astron. Lett. 8, 273 (1982)].

14. D. J. Acheson, Philos. Trans. R. Soc. London, Ser. A 289, 459 (1978).

15. Reviews of Plasma Physics, Ed. by B. B. Kadomtsev (Énergoatomizdat, Moscow, 1984; Consultants Bureau, New York, 1987), Vol. 2.

16. H. C. Spruit, Astron. Astrophys. 349, 189 (1999).

17. Yu. V. Vandakurov, Astron. Zh. 49, 324 (1972) [Sov. Astron. 16, 265 (1972)].

18. R. J. Tayler, Mon. Not. R. Astron. Soc. 161, 365 (1973).

19. M. Stix and D. Skaley, Astron. Astrophys. 232, 234 (1990).

20. M. Watson, Geophys. Astrophys. Fluid Dyn. 16, 285 (1981).

21. S. Chandrasekhar, Hydrodynamic and Hydromagnetic Stability (Clarendon, Oxford, 1961), p. 622.

22. P. A. Gilman and P. A. Fox, Astrophys. J. 484, 439 (1997).

23. M. Goossens, D. Biront, and R. J. Teyler, Astrophys. Space Sci. 75, 521 (1981).

24. L. L. Kitchatinov and G. Rüdiger, Astron. Astrophys. 478, 1 (2008).

25. M. S. Longuet-Higgins, Proc. R. Soc. London, Ser. A 279, 446 (1964).

26. J. Papaloizou and J. E. Pringle, Mon. Not. R. Astron. Soc. 182, 423 (1978).

27. S. I. Vă̌inshtĕn, Ya. B. Zel'dovich, and A. A. Ruzmaikin, Turbulent Dynamo in Astrophysics (Nauka, Moscow, 1980), p. 7 [in Russian].

28. P. Charbonneau, M. Dikpati, and P. A. Gilman, Astrophys. J. 526, 523 (1999).

29. H. C. Spruit, Astron. Astrophys. 381, 923 (2002).

30. H. C. Spruit, in The Internal Solar Angular Velocity, Ed. by B. R. Durney and S. Sofia (Reidel, Dordrecht, 1987), p. 185.

31. L. L. Kitchatinov and G. Rüdiger, Astron. Astrophys. 453, 329 (2006).

Translated by A. Getling 